\title{
Duration of breastfeeding and its determinants in Bangladesh
}

\author{
Mazumder $\mathrm{MS}^{1,2}$ and Hossain $\mathrm{MK}^{3}$ \\ ${ }^{1}$ Department of Agricultural Statistics, Sylhet Agricultural University, Sylhet-3100, ${ }^{2}$ Ph.D Research Fellow \& \\ ${ }^{3}$ Department of Statistics, ShahJalal University of Science and Technology, Sylhet-3114, Bangladesh.
}

[Received: January 16, Accepted: April 30, 2012]

\begin{abstract}
The purpose of this study is to assess the duration of breastfeeding and the factors influencing the duration of breastfeeding in Bangladesh. A total of 4023 mother-child pairs were selected in the study from Bangladesh Demographic and Health Survey (BDHS) 2004. Survival analysis technique (Kaplan Meier, Life table and Cox proportional Hazards model) was employed to investigate the duration and predictor of breastfeeding. The observed mean and median durations of breast-feeding were 30.41 and 35.00 months respectively. Independent effects each of the study variables on duration of breastfeeding were estimated by using Cox's regression model which reveals that the duration of breastfeeding was positively associated with Place of residence, religion of mother and was negatively associated with age at marriage, delivery status, wealth index and maternal education. Younger mothers, mothers from Chittagong and Sylhet division, mothers and having husbands with secondary and higher education, urban mothers, caesarean-section births and Muslim mother were associated with lower duration of breastfeeding. Mothers should be informed about the maternal health benefit of breastfeeding including lactational amenorrhoea, which increases birth interval and maintains good health of the mothers.
\end{abstract}

Key words: Breastfeeding, Cox’s regression, socio-demographic, duration, Bangladesh.

\section{INTRODUCTION}

Breastfeeding is a cost effective way of feeding an infant, providing nourishment for a child at a small cost to the mother. Frequent and exclusive breastfeeding can delay the return of fertility through lactational amenorrhea, though breastfeeding is an imperfect means of birth control. During breastfeeding beneficial hormones are released into the mother's body ${ }^{[1]}$ and the maternal bond can be strengthened ${ }^{[2]}$.

Breastfeeding is the feeding of an infant or young child with breast milk directly from female human breasts (i.e., via lactation) rather than from a baby bottle or other container. Babies have a sucking reflex that enables them to suck and swallow milk ${ }^{[3]}$. Bangladesh has a strong breastfeeding culture. Almost all children are breastfed and continue to be breastfed until they are at least 2 years old. However, sub-optimal practices such as delayed initiation of breastfeeding, prelacteal feeding, non-exclusive breastfeeding, and bottle-feeding are common. Exclusive breastfeeding rates showed little change from 1994 to 2007, ranging between 42 and 46 percent. During this time, some feeding practices improved. e,g. Initiation of breastfeeding within one hour of birth increased from 9 to 24 percent and timely feeding of complementary foods to infants aged 6-9 months rose dramatically, from 28 to 74 percent ${ }^{[4]}$. Figure 1 shows the gaps in exclusive breastfeeding in the first six months and feeding practices at different ages.
Fig 1. Infant and Young Child Feeding (IYCF) Breastfeeding practices in Bangladesh, BDHS 2004, BBF 2005*, BDHS 2007**

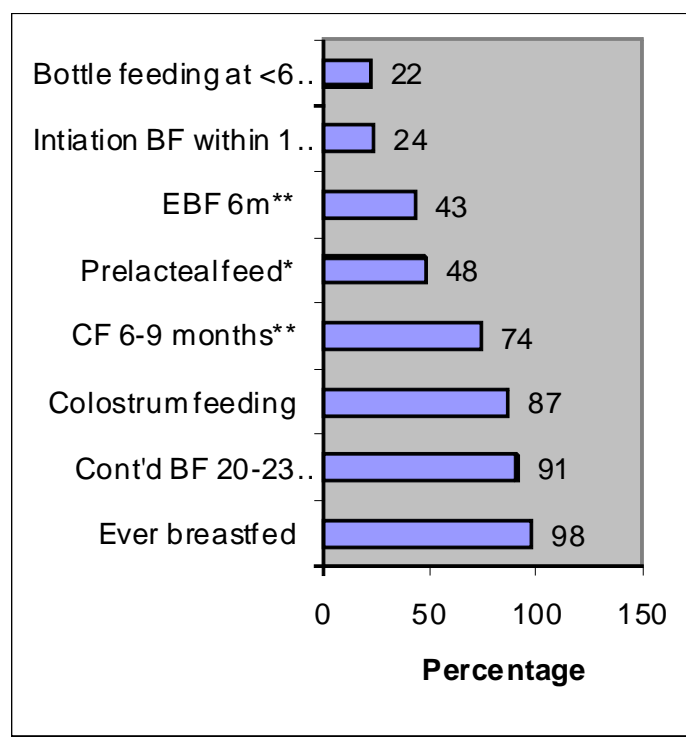

Source: UNICEF 2009.

The present study was undertaken to assess the duration of breast-feeding using survival analysis to identify the cultural, socio- economic and demographic factors influencing the duration of breast-feeding. 


\section{MATERIALS AND METHODS}

Data for the study were drawn from the Bangladesh Demographic and Health Survey (BDHS) 2004. Information was collected on education, age, reproductive behavior, availability of family-planning supplies and services, breastfeeding, child viewed. In total, 4023 mothers provided information on the duration of breastfeeding for their last-born child at the time of interview. Breastfeeding recorded in the survey was 60 months but the study included the duration of breastfeeding up to 36 months and ignored the remaining months as outlier.

Table 1: Mean and medium duration of breastfeeding (in months) by Kaplan Meier \& Life-table method by socio-economic and demographic characteristic, Bangladesh, 2004.

\begin{tabular}{|c|c|c|c|c|}
\hline Characteristics & Number & Percentage & Mean (in months) & $\begin{array}{l}\text { Median (in } \\
\text { months) }\end{array}$ \\
\hline \multicolumn{5}{|c|}{ Age (years) of mothers } \\
\hline $10-19$ & 852 & 21.2 & 30.77 & 35.00 \\
\hline $20-29$ & 2329 & 57.9 & 30.46 & 35.00 \\
\hline 30 and above & 842 & 20.9 & 30.07 & 35.00 \\
\hline \multicolumn{5}{|c|}{ Age (years) at first marriage of mothers } \\
\hline $10-14$ & 1967 & 48.9 & 31.07 & 35.00 \\
\hline $15-19$ & 1764 & 43.8 & 30.16 & 35.00 \\
\hline $20-24$ & 252 & 6.3 & 27.99 & 35.00 \\
\hline 25 and above & 40 & 1.0 & 23.24 & \\
\hline \multicolumn{5}{|l|}{ Delivery status } \\
\hline Normal & 3810 & 94.7 & 30.57 & 35.00 \\
\hline Caesarian & 213 & 5.3 & 26.93 & 34.00 \\
\hline \multicolumn{5}{|c|}{ Region of Residence } \\
\hline Barisal & 425 & 10.6 & 31.31 & 35.00 \\
\hline Chittagong & 897 & 22.3 & 27.98 & 29.34 \\
\hline Dhaka & 866 & 21.5 & 30.38 & 35.00 \\
\hline Khulna & 525 & 13.0 & 31.99 & 35.00 \\
\hline Rajshahi & 745 & 18.5 & 31.52 & 35.00 \\
\hline Sylhet & 565 & 14.0 & 30.26 & 35.00 \\
\hline \multicolumn{5}{|c|}{ Place of Residence } \\
\hline Urban & 1197 & 29.8 & 29.62 & 35.00 \\
\hline Rural & 2826 & 70.2 & 30.78 & 35.00 \\
\hline \multicolumn{5}{|c|}{ Religion of respondent } \\
\hline Muslim & 3681 & 91.5 & 30.27 & 35.00 \\
\hline Non Muslim & 342 & 8.5 & 31.99 & 35.00 \\
\hline \multicolumn{5}{|l|}{ Wealth Index } \\
\hline Poor & 1670 & 41.5 & 30.96 & 35.00 \\
\hline Middle & 787 & 19.6 & 30.72 & 35.00 \\
\hline Rich & 1566 & 38.9 & 29.69 & 35.00 \\
\hline \multicolumn{5}{|c|}{ Education of fathers } \\
\hline No education & 1501 & 37.3 & 31.04 & 35.00 \\
\hline Primary & 1060 & 26.3 & 30.94 & 35.00 \\
\hline Secondary & 1018 & 25.3 & 29.64 & 35.00 \\
\hline Higher & 444 & 11.0 & 28.69 & 35.00 \\
\hline \multicolumn{5}{|c|}{ Education of mothers } \\
\hline No education & 1354 & 33.7 & 30.52 & 35.00 \\
\hline Primary & 1249 & 31.0 & 31.33 & 35.00 \\
\hline Secondary & 1154 & 28.7 & 29.97 & 35.00 \\
\hline Higher & 266 & 6.6 & 27.37 & 30.36 \\
\hline Total & 4023 & 100 & 30.41 & 35.00 \\
\hline
\end{tabular}

Estimation is limited to the largest survival time if it is censored.

health, and maternal status. The survey considered ever-married women of reproductive age as eligible for interview. A sample of 10,500 households was selected from which 11,440 women were inter-
Dependent variable: The duration of breastfeeding is the dependent variable, which was measured in complete months and the variable contained some censored cases. 
The association of the duration of breastfeeding with all the explanatory variables included in this study was first checked by the Chi-square statistic. Chisquare statistic has also been used to test the goodness of fit of the model as well as to test the significance of the explanatory variables.

Both univariate and multivariate proportional hazards model analysis were used to study the duration of breastfeeding. The univariate proportional hazard model analysis was used to give a measure of the effect of each variable on the duration-specific probabilities of the duration of breastfeeding (hazard function) in the absence of the control for other variables. A multivariate proportional hazard model analysis was then undertaken to measure the effect of each category of each variable on the hazard function while controlling the effects of the other variables (and their categories) included in the model. A relative risk of 1.00 was considered to indicate baseline category or reference for each variable. A relative risk greater than 1.00 was taken to indicate higher risks of occurrence of the event than the reference category or vice-versa. Confidence interval $(95 \%)$ of relative risk indicated the level of significance.

Bivariate and multivariate statistical techniques were used for studying the predictor variable - duration of breastfeeding - in relation to the explanatory variables. Survival (Life-table and Kaplan Meier) analysis provides a good understanding of breastfeeding behavior over time. The factors affecting the duration of breastfeeding were investigated in a regression using Cox's proportional hazard model [5]. This model was used for determining the covariates that were significantly associated with the duration of breastfeeding. The hazard ratio (odds ratio) for breastfeeding and its 95\% confidence interval (CI) were calculated for the sociodemographic and cultural factors associated with breastfeeding. The data obtained were analyzed using the SPSS software (version 13.0) and M S Excel.

\section{Duration of breastfeeding}

Breast milk can make the difference between healthy growth and malnutrition, between life and death. When it comes to nutrition, the best first food for babies is breast milk. It has been found that babies given cow's milk or formula by bottle and no breastmilk have over $60 \%$ more risk of being malnourished. Almost 5 million babies each year are at risk of poor nutrition because of inadequate breastfeeding practices in rural areas ${ }^{[6]}$. In our study majority (70\%) lived in rural area and only $30 \%$ lived in urban areas. Maximum (82\%) women got married at adolescent age (10-19 years). More over 91\% were Muslim and only 9\% were non-Muslim (Hindu, Christian and Buddhist).

In this study mean and median duration of breastfeeding of Bangladeshi women under study was 30.4 and 35.0 months respectively (Table 1).
The mean and medium duration of breastfeeding was 28.8 and 32.4 months respectively ${ }^{[7]}$. The mean duration of breastfeeding ${ }^{[8]}$ was found about 26.4 months for the last child.

Mothers who were married at an early age had a longer duration of breastfeeding than those who were married in older age. The average duration of breastfeeding was 30.57 months for mothers who gave birth normally (vaginally) compared to 26.93 months for those who were delivered by caesarian. The mean duration of breastfeeding was almost same in all administrative area except Chittagong (27.98 months) division.

The urban mothers breastfed their children for a relatively shorter duration (29.62 months) than the rural mothers (30.78 months). The non-Muslim mothers breastfed for a longer duration (31.99 months) than the Muslim mothers (30.27 months). In this study, the mean duration of breastfeeding was 30.96, 30.72 and 29.69 months for poor, middle and rich income group women respectively.

Table 2 shows the results (Odds Ratio) of the proportional hazards model for the duration of breastfeeding. Women who got married at an early age ( $\leq 14$ years) had a lower risk of stopping breastfeeding compared to women who were married at an older age (25 years and over). Normal delivery was associated with a $25 \%$ less likelihood of terminating breastfeeding compared to birth by caesarean section. The mothers of Chittagong and Sylhet divisions were more likely to terminate breastfeeding early compared to the mothers in other divisions.

Mothers who lived in urban area were 1.13 times more likely to terminate breast-feeding than the mothers who lived in rural area. The odds of stopping breastfeeding for Muslim mothers were higher (OR 1.97) than the Non-Muslim counterparts. Hazard ratio shows the risk of terminating breastfeeding was lower among mothers with no formal, primary or secondary education than the mothers who had higher education. Risk of terminating breastfeeding was higher among mothers whose husbands had secondary and higher education than those with primary and No education

\section{RESULTS AND DISCUSSION}

The study estimated mean and median duration of breastfeeding of Bangladeshi women are estimated to be 30.4 and 35.0 months respectively. Past studies in Bangladesh obtained average duration of breastfeeding are 28.2, 31.3 and 31.9 months [9-12] respectively for surviving children, which was close to our study. Compared to South Asian countries e.g, India, Pakistan and Sri Lanka where the average durations of breast-feeding were 18.4, 21.8 and 23.2 months ${ }^{[13-15]}$ respectively, where the breast-feeding duration is relatively higher in Bangladesh Age of marriage of women $\leq 14$ years, $15-19$ years and 2024 years were less likely to terminate breastfeeding 
than women who were married at 25 and above years. Mothers from Chittagong and Sylhet were more likely to terminate breastfeeding earlier than those from Barisal, Dhaka, Khulna and Rajahahi division. Muslim mothers had higher risk of terminating of breastfeeding than non-Muslim mothers (Hindu, Christian and Buddhist). The previous study reported non-Muslim mothers breastfeed longer than Muslim mothers in the country ${ }^{[8,9,12,16]}$.

Mothers from rich families had shorter duration of breast-feeding. The reasons might be that economically affluent families could afford to buy powder milk, other baby formulae, wet nurses etc. Figure consciousness among the mothers of higher educated and high-income group also could be a reason for shorter breast-feeding duration.

Mothers should be informed about the value of colostrums and the need for breastfeeding the neonates on demand with special emphasis on the direct positive relationship of higher frequency of suckling and increase in breast milk flow. Also should inform the adverse effects of bottle-feeding and use of tinned milk and milk from sources other than breast milk. Mothers should be informed about the maternal health benefit of breastfeeding including lactational amenorrhoea and its contraceptive effect, which increases birth interval and maintains good health of the mothers.

\section{REFERENCES}

1. $\wedge^{a} b$ "Breastfeeding". Centers for Disease Control andPrevention. http://www.cdc.gov/breastfeeding/. Retrieved 2007-01-23.

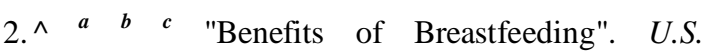
Department of Health and Human Services. http://www.4woman.gov/breastfeeding/index.cfm? page $=227$. Retrieved 2009-02-15.

3. WHO: CDD: 10.2.1992. Indicators for Assessing Breastfeeding Prectices, Bangladesh J Child Health 1991, Vol 15 (3/4) 110-112. Innocents Declaration on the protection, Promotion and Support of Breastfeeding, adopted by participants and the WHO/UNICEF policy makers meeting (co-sponsored by USAID and SIDA), Florence, Italy, 30 July-1 August, 1990.
4. UNICEF (2009). Infat and young child feeding programme review; case study Bangladesh, nutrition section. New York. P-4

5. Lawless JF. (1982) Statistical model and methods for life time data. 2d ed. New York, NY: John Wiley and Sons, 1982:342-400.

6. Robert Parker.; Unicef Beiging 1990.

7. Mitra SN, Al-Sabir A, Islam S, Bhadra SK, Cross A, Kumar S (2005). Bangladesh Demographic and Health Survey, 2004. National Institute of Population Research and Training (NIPORT), Dhaka, Bangladesh

8. Sabina Islam, KNS Yadava and M A Alam (2006). Differentials and determinants of the duration of breastfeeding in Bangladesh: a multilevel analysis. Proc. Pakistan Acad. CciS. 43(1).1-14.

9. Mannan HR, Islam NM. (1995) Breast-feeding in Bangladesh: patterns and impact on fertility. Asia Pac Popul $J$;10:23-38

10.Giashuddin MS, Kabir M. (2003). Breastfeeding duration in Bangladesh and factors associated with it. Indian J Commun Med ;28:34-8.

11.Giashuddin MS, Kabir M. (2004). Duration of breastfeeding in Bangladesh. Indian $J$ Med Res;119:267-72.

12.Akter. S and Rahman. M. (2010). Duration of Breastfeeding and its correlates in Bangladesh. Health nutrition, Dec; 28(6):595-60

13. Rajaretnam (1994). T. Trend and differentials in breast-feeding and amenorrhea durations in a rural area in south India. Demography India; 23 : 83-95.

14.Page HJ, Lesthaeghe RJ, Shah IH (1982). Illustrative analysis: breastfeeding in Pakistan, WFS Scientific Reports No. 37. Voorburg, Netherlands: International Statistical Institute; p. 115 .

15.Mahler K.(1996) Women breast-feeding infants longer in many developing countries. Int Family Planning Persp ; 22 : 134-5.

16.Islam S， Yadava KNS, Alam MA.(2006) Differentials and determinants of the duration of breastfeeding in Bangladesh: a multivariate analysis. Proc Pakistan Acad Sci ;43:1-14. 